\title{
Review
}

\section{Pregnancy associated breast cancer (PABC): Aspects in diagnosis}

\author{
Ioannis Kakoulidis*, Lazaros Skagias and Ekaterini Politi \\ a Postgraduate Program: "Research on Female Reproduction”, Medical School, National \& Kapodistrian \\ University of Athens, Athens, Greece \\ ${ }^{\mathrm{b}}$ Department of Cytopathology, 424 General Army Hospital, Thessaloniki, Greece \\ ${ }^{\mathrm{c}}$ Department of Cytopathology, Aretaieion University Hospital, Athens, Greece
}

\begin{abstract}
Diagnosis of a clinical entity like pregnancy associated breast cancer (PABC) is as demanding and challenging as its rarity. Increasing incidence and controversy that exists in the literature upon prognosis, tumor aggressiveness and underlying mechanisms, highlight the importance of optimizing the diagnostic strategy in women with PABC. Adjustment of standard approach for breast cancer by modifying management methods and options plays key role in decision making. Knowledge of diagnostic modalities and their limitations, in accordance with awareness of physiologic hormone-induced changes of pregnancy and lactation, is the fundamental method of diagnosis in PABC. Thorough triple assessment (history/clinical examination, imaging and cytology/histology) enforces healthcare providers with all essential tools to avoid detrimental delay in diagnosis and to confront with their own hesitation to take action due to limited experience of the disease.
\end{abstract}

Keywords: PABC, pregnancy, breast cancer, diagnosis

\section{Introduction}

Pregnancy-associated breast cancer (PABC) is defined as any breast carcinoma diagnosed during pregnancy or within the first year postpartum [1-4], while some authors estimate the occurence period up to 2 years at any time during lactation [5-7]. The incidence of PABC is increasing [8] at a range of 1:3,000 to $1: 10,000$ pregnancies [3:5-11], with most of the authors referring to $1: 3,000$ ratio approximately [1, 7 11[12], making breast cancer, one of the most frequent types of cancer [7 12] occurring during pregnancy along with melanoma and cervical cancer [2]3, 911]. The average age of women with PABC is between 32 and 38 years of age [37.9], with median age at 34 years [113]. The diagnosis of PABC is expected to become more frequent in the forthcoming years since the percentage of women at first birth

*Corresponding author: Ioannis Kakoulidis, 12 Telesiou Str. Athens 11143, Greece. Tel.: +30 2102528056, +30 6973641971; E-mail: i_kakoulidis@yahoo.gr. aged 35 years and over has increased from $13.2 \%$ to $23.6 \%$ [10], especially in urban areas [5], depicting the increasing trend for women to delay childbearing [4 10 11|14]. Nevertheless, incidence is lower in developing countries as the age of the mother at delivery is younger [415].

There has been a major discussion over the prognosis of PABC, addressed in several studies with inconsistent results [14]. Over the last years, an extreme effort of showing more statistically correct and significant studies has been noticed. Insufficient data on biological features of the tumor and PABC definition [16], along with regional differences, multiple inclusion criteria and deficiency of information in cancer registries about the pregnancy status, emphasize the lack of reliable data [5 17]. The relative rarity of the disease makes impossible the conduction of large powered controlled studies [14] and therefore limited by all of the potential biases [18]. Furthermore, there is a significant controversy in the literature, regarding the role of pregnancy in breast cancer prognosis and PABC outcomes [11]. Many studies do not suggest any signifi- 
cant associations [18-21] whereas other attribute any adverse effects on the delayed diagnosis of tumors in pregnancy [22] or show modest effects [23]. On the contrary, some of them point to the opposite direction, indicating an independent poor prognostic role of pregnancy [22 24-28] with age rather than stage being the most important factor [6]. It has been noticed that breast cancer diagnosed within five years postpartum, has a significant 2.8 times higher risk for metastasis and a 2.7 times higher mortality risk compared to nulliparous cases [2616]. The underlying exact mechanisms that present pregnancy as a poor prognostic factor remain unclear but may be associated with the hormonal environment that characterizes pregnancy [22] and increases breast cancer aggressiveness [29]. On the other hand, poor prognosis may only reflect advanced stage of cancer secondary to delays in diagnosis and management [2 6 14 30]. Breast cancer might be underdiagnosed during pregnancy and lactation [6], likely because of hesitation to carry on with diagnostic procedures [30] due to limited experience in treating this clinical entity [12]. In fact, women with PABC present, more often, with larger tumors and higher incidence of lymph node metastasis at diagnosis. This has been attributed to physiologic changes in pregnancy and lactation that make physical exam difficult [17 25 30]. However, existing data indicate that factors like pregnancy-associated hormones and a delay in diagnosis in recently pregnant women, do not account alone for the high mortality associated with PABC [31].

Medline/PubMed and Google Scholar databases were searched, focusing mainly on clinical and diagnostic aspects of PABC. Reference lists of recently published articles were also searched for additional articles of relevance, yielding a total of 69 English language articles up to November 2014 to be included in this review.

\section{Diagnosis of PABC}

Standard approach for breast cancer might not be suitable during pregnancy [32], since clinical experience of breast cancer in pregnant women is limited, making management decisions inevitably more complicated [12[33]. An adjustment in usual management and a modification of diagnostic methods, therapeutic tools and options is needed [33], in order to avoid possible risk to the fetus [12]. Diagnosis of PABC is based on clinical examination, imaging and histology, simi- lar to non-pregnant women [12], however, it is more difficult due to the major hormone-induced changes that occur in breast tissue, resulting in a delayed, later stage presentation, with more metastases and poorer prognosis [4|12 24|32:34]. This is of critical importance in the management of PABC. Average delay of the diagnosis from the first symptoms ranges from 12 up to 5-7 months. This kind of delay by one month may increase the risk of nodal involvement by $0.9 \%$ to $1.8 \%$ [12]35], while a 6-month delay increases the risk by $5.1 \%$ [8].

Physiologic pregnancy-associated breast changes, including engorgement, hypertrophy and nipple discharge, obscure detection (4) and make physical examination progressively more difficult as pregnancy advances, until they steadily revert to prepregnancy state approximately three months after breastfeeding cessation. Therefore, a careful baseline clinical breast examination at the first obstetric visit is recommended [36]. Breast cancer in pregnancy typically presents as a palpable painless lump [4|34|35|37]. Sometimes it can be found on breast examination in the absence of breastfeeding [35]. Swelling, erythema and diffuse breast enlargement are less common symptoms that suggest locally advanced carcinoma which may act like mastitis [34]. Rarely a bloodstained nipple discharge is observed [12]. During the third trimester of pregnancy, hemoid secretion is often seen and it should be cytologically evaluated if it continues more than 2 months after childbirth [35]. Generally, median duration of symptoms is 5.5 weeks up to 11.5 months [15 37]. Triple assessment (history/clinical examination, imaging and cytology/histology) and awareness of less common presentations of PABC is the fundamental method of accurately diagnosing symptomatic breast complaints in pregnancy [8 12 37]. Especially history taking, including an assessment of risk factors, is crucial. Data from several studies indicate family history as the strongest predictor of breast cancer risk in the female population [3839], High-penetrance genes such as BRCA1 and BRCA2 explain less than $20 \%$ of the inherited risk, the rest being accounted for moderate and low penetrance [40]. Nevertheless, individuals with BRCA1 or BRCA2 mutations might be at increased risk, but it seems that they do not have an increased incidence of PABC [4]. There are only indications that PABC prevalence is higher in cases of BRCA1 mutation [35]. 


\section{Imaging}

Breast imaging during pregnancy requires particular expertise [12]. Due to its rarity and typically unclear clinical findings upon examination, the diagnosis of a contralateral lesion in PABC can be particularly challenging [41]. A tumor mass may appear as a normal tissue or may be located deeper as the breast increases its size, making it more difficult to identify [35].

\subsection{Mammography}

The mammographic features of PABC do not differ from those of non-PABC [42]. Mammographic parenchymal density during pregnancy increases and diffuses, especially during lactation where is further increased so that expansion of the lactic duct seems to be nodular with hyperdensity by breast milk filling nipples [35]. Mammography is able to detect a high proportion of cancers between $63 \%$ and $90 \%$ in most series [17/34/35/37]. Although masses may not be noticeable, typical malignant microcalcifications can be detected even in extremely dense breasts. Furthermore, asymmetric density, axillary lymphadenopathy, diffuse skin and trabecular thickening are also helpful in detecting mammographic abnormalities in these patients [43]. However, in pregnant and lactating women, it is difficult to diagnose a tumor by mammography due to its architectural distortion and asymmetric density [3544]. The sensitivity of conventional mammography is decreasing at $86.7 \%$ due to increased parenchymal density, glandularity and water content of the lactating breast [34/4345]46]. Routine screening mammography is not performed during pregnancy, until three months after cessation of lactation to allow the breast parenchyma to fully involute and return to baseline density [47].

Nevertheless, an important and also controversial issue regarding the use of mammography during pregnancy is the exposure of the developing fetus to ionising radiation [37]. It is thought that fetal abnormalities may occur in case of more than 0.05 Gy $(5 \mathrm{rad})$. Yet, the radiation that fetus can receive through the mammography is very small as $0.004 \mathrm{~Gy}(0.4 \mathrm{mrad})$, even if there is no use of lead aprons [17|35[46]. However, except special cases, current recommendations suggest to avoid mammography during the first trimester of pregnancy and instead use ultrasound [34|35]. The later is preferred regardeless the fact that mammography performed with proper fetal shielding, has been successfully used during pregnancy [17,46], presenting less risk for the fetus [12] and considered to be generally safe during pregnancy and lactation [47]. Appropriate abdominal shielding decreases the dose to the uterus up to $50 \%$, despite the fact that most of the dose is from scatter radiation and shielding might has limited effectiveness. Nevertheless, it must still be offered to all patients [47].

In general, mammography is considered to be essential in cases of suspected or proven by core needle biopsy underlying malignancy [35|4748] or a biopsy is indicated by ultrasonography and further investigation must be performed to identify the range of lesions, including microcalcification lesions [35], The most prominent recommendation, is to start with one oblique view. When a suspicious mass is noticed, both craniocaudal and mediolateral oblique views of both breasts are needed to exclude multicentric and bilateral disease [12]. Digital mammography has been shown to be more sensitive in younger women with dense breast tissue, since it may be associated with an up to $75 \%$ reduction in radiation dose [37]. There is no concern about mammography during lactation [42]. Breast should be emptied as much as possible prior to performing mammography to reduce density [36 37/47].

\subsection{Breast ultrasonography}

Breast ultrasound is the imaging modality of choice during pregnancy and lactation due to lack of ionising radiation and widespread availability [34 3747]. In most series, breast ultrasound is between $93 \%$ to $100 \%$ sensitive in demonstrating abnormalities in symptomatic women [35]37/43] and has 100\% negative predictive value for PABC [47]. Its high sensitivity and specificity in diagnosis of PABC, due to its ability to distinguish between cystic and solid breast lesions, emerges ultrasound as the standard method for the evaluation of any palpable breast mass or other lesion characteristics during pregnancy [12 17/37/46]. Imaging both breasts primarily is important as the incidence of bilateral disease may be as high as $10 \%$. Multicentric and multifocal cancer has been identified in between $14 \%$ and $47 \%$ of cases [37]. Approximately $90 \%$ of women with PABC present with breast masses, easily evaluated with ultrasound.

The most commonly described sonographic finding in PABC is a mass with irregular shape and ill defined margins, with predominantly hypoechoic rather than mixed echogenicity. During pregnancy, the breast parenchyma is characterized by enlargement of the non fatty fibroglandular component with slight 
diffuse hypoechogenicity. In contrast, during lactation, the parenchyma shows diffuse hyperechogenicity, a prominent ductal system and increased vascularity [34 35 37/47/49]. In both pregnancy and lactation, hypervascularity of the breast tissue can be observed. After cessation of breastfeeding, the sonographic appearance of the breast returns to the pre-pregnancy state [36]. In case of malignant breast lumps, ultrasonography is more sensitive than mammography [35]. However, physiological changes associated with the breast and lactation may alter typical sonographic features of breast carcinoma [42]. Parallel orientation has been reported in 58\% of PABC. Posterior acoustic enhancement, characteristic of large or superficial breast cysts and commonly seen in benign breast lesions, has also been noticed in $63 \%$ of PABC cases [43]. Ahn et al. showed that $30 \%$ of the patients with posterior enhancement had complex echo patterns with large cystic components. Cystic appearance with posterior acoustic enhancement is likely to depict the overgrowth of vascular supply with necrosis as a result of stimulation by the hormonal changes associated with pregnancy and may reflect the aggressive nature of tumors in PABC [35[43].

\subsection{Magnetic resonance imaging}

Breast magnetic resonance imaging (MRI) is useful in staging of breast cancer in non-pregnant women, by detecting breast cancer and its metastases to liver, bone and brain [17], in assessing response to neo-adjuvant chemotherapy [37], and in depicting disease extent and multicentricity [42]. However, the usefulness, accuracy and safety of MRI during pregnancy and lactation are still being under consideration [4|37]. The routine use of MRI in the evaluation and treatment of pregnant patients is not recommended, except if only in situations where the risk-benefit ratio is clear [34|42], such as if the examination provides important information that would otherwise require exposure to ionising radiation [12 35 48] . Furthermore, MRI assessment might be difficult and controversial during lactation, due to rapid enhancement of lactational breast parenchyma after intravenous administration of contrast media, followed by an early plateau of enhancement. However, due to increased fraction of mobile water in milk, lactational parenchyma has diffuse high signal intensity on T2-weighted images and therefore more reliable visualization of tumors [34]. The observed MRI features of cancer in lactating breast tissue include lower signal intensity compared with the surrounding lactating glandular tissue on T2-weighted images, strong rapid initial contrast enhancement with early washout, irregular margins and occasional rim enhancement [45].

Generally, except special cases, contrast media use, such as gadolinium for imaging enhancement [37], is not recommended [35]. No results of breast MRI specificity and sensitivity in pregnant patients have yet been reported [4[12], and there are no known recorded adverse effects following MRI or gadolinium administration during pregnancy. Still, there are concerns regarding exposure of the fetus to high magnetic fields and the long-term effects of exposure to free gadolinium ions [39] based on studies in animal models showing that gadolinium crosses the placenta and is associated with fetal abnormalities [17/46]. Gadolinium is associated with nephrogenic systemic fibrosis in adults with an impaired kidney function. However, children under 1 year are considered at low-risk because of their immature renal function [412]. Furthermore, some studies report that contrast media might not be closely related to fetal abnormalities and that gadolinium based contrast could be safely utilized during pregnancy [35]. Nevertheless, MRI should be used with caution, especially during the first trimester. If needed, Gadobenate dimeglumine and Gadoterate meglumine contrast media could be used, since no unconfounded cases of nephrogenic systemic fibrosis have been reported [4]12]. On the other hand, as in non-pregnant cases, screening the bones is not necessary in stage I and II tumors [17], so when the estimated risk of metastatic disease is low, postponement of staging until after delivery can be considered [4]. Contrastenhanced MRI may be resumed eventually during lactation [4142] in patients who are at very high risk for breast cancer [47]. Gadolinium use in MRI during lactation is known to be relatively safe [35], as gadolinium-based agents are excreted into breast milk at a rate of $0.0004 \%$ of the maternal dose. However, in order to avoid any ingestion of gadolinium by the infant, breast milk should be pumped and discarded for 24 hours after the administration [41].

Lungs, bone and liver are the commonest metastatic sites and should undergo staging procedures including chest X-ray, liver ultrasound, bone scanning or MRI if the risk of metastasis is sufficiently high. In low risk cases, staging should be postponed to after delivery. Chest radiography, for detecting pulmonary metastasis, can be carried out safely during pregnancy with abdominal shielding. Liver ultrasound is the preferred technique to detect liver metastases. MRI could be used additionally to detect bone metastasis. Bone scan is only recommended in cases of uncertain MRI findings, or MRI unavailability [12]. 


\section{Cytology}

\subsection{Core biopsy}

Knowledge of the tumor grade and subtype is crucial for the management and treatment of breast cancer. Any new palpable mass found during pregnancy and lactation that does not resolve within 2 weeks should be carefully investigated further [12|37|46|50]. Biopsy of a suspicious mass is the most prominent method to obtain histological diagnosis of breast cancer and core needle biopsy is the technique of choice. Its sensitivity is around 90\% [4|12], emerging as the standard safe, cost-effective and easy procedure for assessing breast masses during pregnancy and lactation, making precise diagnosis and avoiding surgical biopsy [34]. It has many advantages over fine needle aspiration (FNA), as it provides specific information to tumor type, grade and receptor status, facilitating treatment planning and patient counseling [48]. Furthermore, it does not require cytology expertise and reduces the incidence of unsatisfactory or insufficient results [37]. Ultrasoundguided core needle biopsy is the most preferable procedure. Stereotactic biopsy and mammography-guided wire localization are safe and feasible during early pregnancy and can be performed in cases of ultrasound insufficiency. MRI-guided procedures can be utilized in lactating women [47]. Imaging and biopsies can be performed at a single session as appropriate [46].

Despite the fact that core biopsy is a safe technique, there is still a considerable risk of bleeding, due to increased vascularity associated with pregnancy and lactation. The risk of infection is also increased attributed to ductal dilatation, milk production and breastfeeding traumas, as is the risk of milk fistula formation that occurs more in central than in peripheral [34|35|37]. These complications occur rarely and are less likely to develop with FNA. Risk can be minimized by pausing breastfeeding before undergoing biopsy, attentive hemostasis and procedure performance with strict asepsia [34[35]. Furthermore, the use of $14 \mathrm{G}$ needle caliper rather than $9 \mathrm{G}$ or $10 \mathrm{G}$, could reduce the risk of galactocele or milk fistula formation [37].

\subsection{Fine needle aspiration}

Fine needle aspiration cytology (FNAC) of the breast is becoming an increasingly important diagnostic tool in the assessment of breast malignancies and their further subtyping with a high degree of accuracy [50[51]. It has been shown to be rapid, minimally invasive and safe diagnostic procedure in pregnancy and lactation, indicating suspicious or malignant findings in $95 \%$ of lesions [48,50-52]. ThinPrep Papanicolaou test is considered to be a trusted and widely used technique, much easier and faster as procedure. However, it has not fully replaced conventional smears because of the cytological artifacts of rapid fixation, such as decreased cell cluster size and the fragmentation of cellular sheets and individual cells [53]. The established diagnosis of breast cancer by FNA allows more flexibility for treatment planning providing prognostic information [50]. In most cases the diagnosis is based on the findings from FNA or core biopsy, so it is important that the pathologist is aware of the pregnant or lactational state of the breast, in order to avoid overdiagnosis so that a cancer is not assumed to be due to pregnancy related atypia. In fact, FNAC can be difficult to interpret in pregnant and lactating patients as cellular atypia can be also seen in disease-free women [12[37/48]. Therefore, FNA should be initially performed in cases where a skilled cytopathologist is available. Otherwise core biopsy should be considered, especially if the lesion is calcified or cytology results are inconclusive or discordant with imaging or clinical findings [35 37]. Whenever biopsies or surgical specimens are not available, immunocytochemical analysis of FNA material, with either cell block or ThinPrep techniques, is a reliable method for the estrogen receptor (ER) and progesterone receptor (PR) status determination. Performed under strict conditions using primary breast carcinomas, it can become helpful in metastatic settings [53].

Furthermore, a growing area of research is related to the use of computational intelligence based techniques applied to the processing of information necessary for the diagnosis in FNA. Sizilio et al introduced a method to assist in the diagnosis and second opinion of breast cancer from the analysis of descriptors extracted from smears of breast mass obtained by FNA, with the use of computational intelligence resources, so called fuzzy logic. The Fuzzy Method was capable of providing breast cancer pre-diagnosis with $98.59 \%$ sensitivity and $85.43 \%$ specificity [54].

\subsection{Ultrasound guided FNA}

Generally, breast imaging techniques can not detect all breast cancers, therefore any discrete palpable breast mass or clinical finding not explained following ultrasound should undergo direct FNA [48]. Because of the overlapping sonographic features of 
benign and suspicious metastatic lymph nodes, ultrasound was combined with FNAC for more accurate results. Ultrasound-guided FNA is a highly specific technique for detecting axillary metastases in breast cancer. Its sensitivity is $89 \%$, specificity $100 \%$ and accuracy $90.4 \%$ respectively [55] and has been reported to increase the sensitivity of preoperative nodal staging [56 57], providing a more simple, minimally invasive and reliable approach to the initial determination. When ultrasound-guided FNAC is positive, an axillary lymph node dissection can be performed, without sentinel biopsy [56]. Furthermore, the adoption of routine preoperative axillary sonography with FNA is a lowercost strategy especially in patients with stage $>\mathrm{T} 2$ invasive breast cancer [57].

\subsection{Sensitivity and false positive or negative results}

Since mammary epithelial cells undergo several cellular morphological changes during pregnancy and lactation [35], FNA may be associated with increased false-positive and false-negative rates [7]. Therefore, the results must be carefully assessed [35], due to certain limitations of FNAC which require final histopathological diagnosis for establishing prognosis and therapy. The key point is to determine the diagnostic accuracy of FNA cytology and to develop distinguishing cytologic criteria addressing the diagnostic challenges [51]. Karim et al. showed in rats an FNA sensitivity of $92.3 \%$ and specificity of $89.4 \%$ for the detection of malignancy in comparison with histologic diagnosis of tissue biopsies. However, $14 \%$ of specimens, all fibroadenomas by histology, had insufficient cells for cytologic evaluation, for an overall accuracy rate of $78.0 \%$. Malignant tumors had significantly higher scores for all cytomorphologic features and were significantly more likely to contain cell clusters and necrotic debris. Most false positive results were related to the presence of atypical single epithelial cells in fibroadenomas, and some false negative were solid carcinomas by histologic examination. The low cellularity and clean background led to misdiagnosis of the solid carcinomas as benign rather than malignant [52]. In addition, care must be taken when squamous cells are seen on FNA, while a broad differential diagnosis should be considered [58]. Sometimes apocrine metaplasia may be confused with squamous cells due to their polygonal appearance and dense eosinophilic cytoplasm. Careful assessment of cytological features of squamous cells and the appearance of the background is crucial for achieving a correct di- agnosis. Deep knowledge of the cytomorphology and cytodiagnosis of rare breast malignant tumors is crucial in the FNA analysis [51].

\subsection{FNA biomarkers - Genomic analysis - Immunohistochemistry}

The use of cytological specimens to evaluate tumor biomarkers in metastatic breast cancer lesions has attracted increased interest due to considerable number of reports showing discordance between the primary tumor and metastatic lesion [53]. Russo et al. showed data that demonstrate the capability to perform cDNAarray in RNA extracted from FNA specimen and provide evidence that RNA can be obtained from human buccal mucosa and serum. Moreover, the gene expression profiles obtained from these sources detected the same genes which are overexpressed in the parous breast epithelia [59]. There have been also attempts to develop epigenetic-based markers for breast cancer detection in clinical samples obtained by FNA, nipple aspiration or ductal lavage fluid. Comprehensive breast cancer methylation profiling might provide biomarkers for diagnostic assessment of suspicious breast lesions using FNA, despite the fact that the use of conventional methylation specific polymerase chain reaction (MSP) in most of the studies limited the usefulness of those markers due to the detection of significant gene promoter methylation in normal breast tissue [60].

Hormone receptor expression, on the other hand, is a well known prognostic and predictive biomarker. The detection method has developed over time from different biochemical assays to immunohistochemistry. The later is a rapid, cost effective, reliable and easy method. The analysis is suitable even for very small tumors and needle biopsy specimen. Immunohistochemistry can distinguish between normal epithelial cells, invasive tumor cells and in situ lesions, giving a more precise result. However, data show that biochemical assays is a more sensitive method than immunohistochemistry in detecting ER positivity [61]. Nevertheless, some tumors may be falsely classified as ER and PR negative due to downregulation of the receptors related to high circulating estrogen and progesterone levels [8]. Ligand dependent ER/PR downregulation is a well described phenomenon that paradoxically is a sign of active functional transcription involving the receptors [62]. Up to $20 \%$ of current immunohistochemistry ER/PR determinations testing worldwide may be inaccurate. Most testing issues have occurred due to variation in preanalytic variables, thresholds for posi- 
tivity and interpretation criteria. Therefore, it is recommended that ER and PR assays may be considered positive if there are at least $1 \%$ positive tumor nuclei in the sample on testing in the presence of expected reactivity of internal (normal epithelial elements) and external controls [63]. In addition, by removing first unbound circulating estrogen by dextran charcoal may also improve the accuracy of determination of hormone receptor expression and decrease the potential risk of false negativity [8].

\subsection{Nipple discharge smear test-Breast milk}

Nipple discharge is the third most common breast complaint after breast pain and breast mass, generally associated with endocrine alterations or medications, pregnancy, lactation and benign or malignant breast lessions. It can be of several types of composition and density. Cytological examination of nipple discharge is a challenging noninvasive method of diagnosing the underlying breast pathology and very helpful in detecting a breast lesion even if there is no palpable mass in the breast, making nipple discharge the only specimen available for cytodiagnosis in these cases [64]. Nipple discharge cytology is as specific as FNAC but less sensitive, ranging from $40 \%$ to $70 \%$, with a mean detection of $66 \%$ and $0.9 \%$ to $2.6 \%$ false positive rate. Nevertheless, it can be a cost effective and valuable tool in the evaluation of abnormal nipple discharge [6465]. Furthermore, breast milk could also serve as a noninvasive source for studying cells of the mature gland and the pregnancy-lactation cycle. Plentiful in growth factors, chemokines, and immunomodulatory, anti-inflammatory and proinflammatory mediators, breast milk also contains potentially stem-like or progenitor cells. Several of these factors have important roles in breast development and breast cancer, and therefore, additional research is needed to assess whether DNA methylation of tumor suppressor genes in milk is associated with breast cancer risk [66].

\subsection{Sentinel lymph node biopsy-Lymphatic mapping}

Axillary lymph node status is an important factor in prognosis assessment and treatment management in invasive breast cancer. Sentinel lymph node biopsy (SLNB) has replaced axillary lymph node dissection (ALND) to stage the axilla in clinically node negative breast cancer patients [55|67]. It can be considered in multicentric/multifocal breast cancer, but it should be employed cautiously in cases of large tumor burdens due to the higher false-negative rate observed [55]. A careful ultrasound assessment of the axilla with FNA in patients presenting with invasive breast cancer is a highly specific and valuable tool in assessing suitability for SLNB. It prevents unnecessary axillary clearance since it can spare a SLNB in $17 \%$ to $20 \%$ of cases, and significantly reduces the incidence of a second axillary procedure after SLNB [6768]. Patients in whom axillary involvement is diagnosed by ultrasound-guided FNA have significantly more positive nodes compared to sentinel lymph node positive patients resulting in a sensitivity of $89 \%$, specificity of $100 \%$ and accuracy of $90.4 \%$ [68].

SLNB can be performed safely during pregnancy using isosulfan blue dye alone, as the use of a radioisotope could be harmful for the fetus. However, even blue dye might be related to adverse effects due to a possible risk of an anaphylactic maternal reaction and therefore, its use is debated [12]. Eventually, concerns regarding isosulfan blue traversing the placenta and radiation exposure during SLNB, underline the suitability of axillary dissection. This fact is likely to change based on the knowledge that the radiation dose to the fetus associated with SLNB mapping is minimal [37]. Keleher et al, based on a theoretical model, found that the maximum absorbed dose to the fetus in pregnant women undergoing breast lymphoscintigraphy with $92.5 \mathrm{MBq}$ (2.5 mCi) of Tc-99m sulfur colloid is $4.3 \mathrm{mGy}$. The threshold absorbed dose for adverse effect is being estimated between $50 \mathrm{mGy}$ and $110 \mathrm{mGy}$. The risk to the fetus is small enough, that breast lymphoscintigraphy followed by SLNB should be evaluated as an alternative to complete axillary lymph node dissection in PABC [69]. If needed it is advisable to inject Tc$99 \mathrm{~m}$ sulfur colloid in the morning to reduce the time and dose of radiation [12]. However, further studies are essential to address both the efficiency of the placenta in excluding the radiopharmaceutical from the fetal circulation and the long-term effects in child and mother [69].

\section{Conclusions}

Diagnosis of a clinical entity like pregnancy associated breast cancer (PABC) is as demanding and challenging as its rarity. Increasing incidence and controversy that exists in the literature upon prognosis, tumor aggressiveness and underlying mechanisms, highlight the importance of optimizing the diagnostic strat- 
egy in women with PABC. Adjustment of standard approach for breast cancer by modifying management methods and options plays key role in decision making. Knowledge of diagnostic modalities and their limitations, in accordance with awareness of physiologic hormone-induced changes of pregnancy and lactation, is the fundamental method of diagnosis in PABC. Thorough triple assessment (history/clinical examination, imaging and cytology/histology) enforces healthcare providers with all essential tools to avoid detrimental delay in diagnosis and to confront with their own hesitation to take action due to limited experience of the disease. Breast ultrasound is the first diagnostic tool used when a breast mass and the axillary area need to be assessed during pregnancy. When breast cancer is diagnosed, bilateral and multicentric disease can be excluded with mammography. MRI with contrast agents is possible during pregnancy, but should only be used when it will alter clinical decision making or when other imaging modalities are insufficient. Ultrasoundguided FNA should be used primarily in cases where a skilled cytopathologist is available. Otherwise, core biopsy should be considered, especially when cytology results are inconclusive or discordant with imaging or clinical findings. Further research is essential to address important matters related to sensitivity and safety of diagnostic procedures in PABC.

\section{Conflict of interest}

The authors declare that they have no conflict of interest.

\section{References}

[1] N.A. Pavlidis, Coexistence of Pregnancy and Malignancy, Oncologist 7 (2002), 279-287.

[2] I. Navrozoglou, T. Vrekoussis, E. Kontostolis, V. Dousias, S. Zervoudis, E.N. Stathopoulos, O. Zoras and E. Paraskevaidis, Breast cancer during pregnancy: A mini-review, Eur J Surg Oncol 34 (2008), 837-843.

[3] A. Molckovsky and Y. Madarnas, Breast cancer in pregnancy: A literature review, Breast Cancer Res Treat 108 (2008), 333338.

[4] F. Amant, S. Loibl, P. Neven and K. Van Calsteren, Breast cancer in pregnancy, Lancet 379 (2012), 570-79.

[5] T.M. Andersson, A.L. Johansson, C.C. Hsieh, S. Cnattingius and M. Lambe, Increasing Incidence of PregnancyAssociated Breast Cancer in Sweden, Obstetrics \& Gynecology 114 (2009), 568-572.

[6] A.L. Johansson, T.M. Andersson, C.C. Hsieh, K. Jirström, P. Dickman, S. Cnattingius and M. Lambe, Stage at diagnosis and mortality in women with pregnancy-associated breast cancer (PABC), Breast Cancer Res Treat 139 (2013), 183192.
[7] I.A. Jacobs, C.K. Chang and G.I. Salti, Coexistence of Pregnancy and Cancer, Am Surg 70 (2004), 1025-1029.

[8] K.S. Asgeirsson, Pregnancy-associated breast cancer, Acta Obstet Gynecol Scand 90 (2011), 158-166.

[9] B. Bodner-Adler, K. Bonder and H. Zeisler, Breast cancer diagnosed during pregnancy, Anticancer Res 27(3B) (2007), 1705-1708.

[10] Y. Lee, C. Roberts, T. Dobbins, E. Stavrou, K. Black, J. Morris and J. Young, Incidence and outcomes of pregnancyassociated cancer in Australia, 1994-2008: A populationbased linkage study, BJOG 119 (2012), 1572-1582.

[11] F. Zagouri, T. Psaltopoulou, C. Dimitrakakis, R. Bartsch and $\mathrm{M}-\mathrm{A}$. Dimopoulos, Challenges in managing breast cancer during pregnancy, J Thorac Dis 5(S1) (2013), S62-67.

[12] F. Amant, S. Deckers, K. Van Calsteren, S. Loibl, M. Halaska, L. Brepoels, J. Beijnen, F. Cardoso, O. Gentilini, L. Lagae, O. Mir, P. Neven, N. Ottevanger, S. Pans, F. Peccatori, R. Rouzier, H-J. Senn, H. Struikmans, M-R. Christiaens, D. Cameron and A. Du Bois, Breast cancer in pregnancy: Recommendations of an international consensus meeting, Eur J Cancer 46 (2010), 3158-3168.

[13] J.L. Meisel, K.E. Economy, K.Z. Calvillo, L. Schapira, N.M. Tung, S. Gelber, S. Kereakoglow, A.H. Partridge and E.L. Mayer, Contemporary multidisciplinary treatment of pregnancy-associated breast cancer, Springerplus 2 (2013), 297.

[14] H.A. Azim Jr, E. Botteri, G. Renne, P. Dell'orto, N. Rotmensz, O. Gentilini, C. Sangalli, G. Pruneri, B. Di Nubila, M. Locatelli, C. Sotiriou, M. Piccart, A. Goldhirsch, G. Viale and F.A. Peccatori, The biological features and prognosis of breast cancer diagnosed during pregnancy: A case-control study, Acta Oncol 51 (2012), 653-661.

[15] A. Gogia, S. Deo, N.K. Shukla, B.K. Mohanti and V. Raina, Pregnancy associated breast cancer: An institutional experience, Indian J Cancer 51 (2014), 167-169.

[16] E.B. Callihan, D. Gao, S. Jindal, T.R. Lyons, E. Manthey, S. Edgerton, A. Urquhart, P. Schedin and V.F. Borges, Postpartum diagnosis demonstrates a high risk for metastasis and merits an expanded definition of pregnancy-associated breast cancer, Breast Cancer Res Treat 138 (2013), 549-559.

[17] E. Doğer, E. Çalişkan and P. Mallmann, Pregnancy associated breast cancer and pregnancy after breast cancer treatment, $J$ Turk Ger Gynecol Assoc 12 (2011), 247-55.

[18] C.G. Murphy, D. Mallam, S. Stein, S. Patil, J. Howard, N. Sklarin, C.A. Hudis, M.L. Gemignani and A.D. Seidman, Current or recent pregnancy is associated with adverse pathologic features but not impaired survival in early breast cancer, Cancer 118 (2012), 3254-3259.

[19] F. Amant, G. von Minckwitz, S.N. Han, M. Bontenbal, E. Ring Alistair, J. Giermek, H. Wildiers, T. Fehm, S.C. Linn, B. Schlehe, P. Neven, P.J. Westenend, V. Müller, K. Van Calsteren, B. Rack, V. Nekljudova, N. Harbeck, M. Untch, P.O. Witteveen, K. Schwedler, C. Thomssen, B. Van Calster and S. Loibl, Prognosis of Women With Primary Breast Cancer Diagnosed During Pregnancy: Results From an International Collaborative Study, J Clin Oncol 31 (2013), 2532-2539.

[20] M.J. Halaska, G. Pentheroudakis, P. Strnad, H. Stankusova, J. Chod, H. Robova, L. Petruzelka, L. Rob and N. Pavlidis, Presentation, Management and Outcome of 32 Patients with Pregnancy-Associated Breast Cancer: A Matched Controlled Study, Breast J 15 (2009), 461-467.

[21] C. Sánchez, F. Acevedo, L. Medina, C. Ibáñez, D. Razmilic, M.E. Navarro and M. Camus, Breast cancer and pregnancy: 
A comparative analysis of a Chilean cohort, Ecancermedicalscience 8 (2014), 434

[22] C. Dimitrakakis, F. Zagouri, A. Tsigginou, S. Marinopoulos, T.N. Sergentanis, A. Keramopoulos, G.C. Zografos, K. Ampela, D. Mpaltas, C. Papadimitriou, M.A. Dimopoulos and A. Antsaklis, Does Pregnancy-Associated Breast Cancer Imply a Worse Prognosis? A Matched Case-Case Study, Breast Care 8 (2013), 203-207.

[23] A.O. Rodriguez, H. Chew, R. Cress, G. Xing, S. McElvy, B. Danielsen and L. Smith, Evidence of Poorer Survival in Pregnancy-Associated Breast Cancer, Obstet Gynecol 112 (2008), 71-78.

[24] H.A. Azim Jr, L. Santoro, W. Russell-Edu, G. Pentheroudakis, N. Pavlidis and F.A. Peccatori, Prognosis of pregnancyassociated breast cancer: A meta-analysis of 30 studies, Cancer Treat Rev 38 (2012), 834-842.

[25] S.A. Ali, S. Gupta, R. Sehgal and V. Vogel, Survival Outcomes in Pregnancy Associated Breast Cancer: A Retrospective Case Control Study, Breast J 18 (2012), 139-144.

[26] W.B. Moreira, E.C. Brandão, A.N. Soares, C.E. Lucena and C.M. Antunes, Prognosis for patients diagnosed with pregnancy-associated breast cancer: A paired case-control study, Sao Paulo Med J 128 (2010), 119-124.

[27] A.L. Johansson, T.M. Andersson, C.C. Hsieh, S. Cnattingius and M. Lambe, Increased mortality in women with breast cancer detected during pregnancy and different periods postpartum, Cancer Epidemiol Biomarkers Prev 20 (2011), 18651872.

[28] W. Reed, B. Sandstad, R. Holm and J.M. Nesland, The Prognostic Impact of Hormone Receptors and c-erbB-2 in Pregnancy-Associated Breast Cancer and Their Correlation with BRCAl and Cell Cycle Modulators, Int J Surg Pathol 11 (2003), 65-74

[29] C. Mathelin, K. Annane, A. Treisser, M.P. Chenard, C. Tomasetto, J.P. Bellocq and M.C. Rio, Pregnancy and postpartum breast cancer: A prospective study, Anticancer Res $\mathbf{2 8}$ (2008), 2447-2452.

[30] L.A. Buré, L. Azoulay, A. Benjamin and H.A. Abenhaim, Pregnancy-Associated Breast Cancer: A Review for the Obstetrical Care Provider, J Obstet Gynaecol Can 33 (2011), 330-337.

[31] P. Schedin, Pregnancy-associated breast cancer and metastasis, Nat Rev Cancer 6 (2006), 281-291.

[32] F. Hoellen, R. Reibke, K. Hornemann, M. Thill, D.W. Luedders, K. Kelling, A. Hornemann and M.K. Bohlmann, Cancer in pregnancy. Part II: treatment options of breast and other non-gynecological malignancies, Arch Gynecol Obstet 284 (2011), 1481-1494.

[33] M.C. Sánchez and S.A. Ruiz, Breast cancer during pregnancy, Breast Cancer Res Treat 123(Suppl1) (2010), 55-58.

[34] J.M. Sabate, M. Clotet, S. Torrubia, A. Gomez, R. Guerrero, P. de las Heras and E. Lerma, Radiologic Evaluation of Breast Disorders Related to Pregnancy and Lactation, Radiographics 27(Suppl1) (2007), 101-124.

[35] J.H. Yu, M.J. Kim, H. Cho, H.J. Liu, S.J. Han and T.G. Ahn, Breast diseases during pregnancy and lactation, Obstet Gynecol Sci 56 (2013), 143-159.

[36] R. Vashi, R. Hooley, R. Butler, J. Geisel and L. Philpotts, Breast imaging of the pregnant and lactating patient: Physiologic changes and common benign entities, AJR AM J Roentgenol 200 (2013), 329-336.

[37] C.M. Saunders, D. Taylor and A. Ives, The role of breast imaging during pregnancy and lactation in the diagnosis of breast malignancy, The Radiographer 59 (2012), 119-123.
[38] A. Hadjisavvas, M.A. Loizidou, N. Middleton, T. Michael, R. Papachristoforou, E. Kakouri, M. Daniel, P. Papadopoulos, S. Malas, Y. Marcou and K. Kyriacou, An investigation of breast cancer risk factors in Cyprus: A case control study, BMC Cancer 10 (2010), 447.

[39] P. This, A. de la Rochefordiére, A. Savignoni, M.C. Falcou, A. Tardivon, F. Thibault, S. Alran, V. Fourchotte, A. Fitoussi, B. Couturaud, S. Dolbeault, R.J. Salmon, B. Sigal-Zafrani, B. Asselain and D. Stoppa-Lyonnet, Breast and ovarian cancer risk management in a French cohort of 158 women carrying a BRCA1 or BRCA2 germline mutation: Patient choices and outcome, Fam Cancer 11 (2012), 473-482.

[40] M. Pollán, Epidemiology of breast cancer in young women, Breast Cancer Res Treat 123 (2010), 3-6.

[41] T. Fujita, N. Taira, Y. Ogasawara, M. Omori and H. Doihara, Bilateral angiosarcoma of the breast detected by magnetic resonance imaging during pregnancy, Int J Clin Oncol 14 (2009), 560-563.

[42] A.P. Ayyappan, S. Kulkarni and P. Crystal, Pregnancyassociated breast cancer: spectrum of imaging appearances, Br J Radiol 83 (2010), 529-534.

[43] B.Y. Ahn, H.H. Kim, W.K. Moon, E.D. Pisano, H.S. Kim, E.S. Cha, J.S. Kim, K.K. Oh and S.H. Park, Pregnancy- and lactation-associated breast cancer: Mammographic and sonographic findings, J Ultrasound Med 22 (2003), 491-497.

[44] P. Eedarapalli and S. Jain, Breast cancer in pregnancy, $\mathrm{J} \mathrm{Ob}$ stet Gynaecol 26 (2006), 1-4.

[45] L.A. Espinosa, B.L. Daniel, L. Vidarsson, M. Zakhour, D.M. Ikeda and R.J. Herfkens, The lactating breast: Contrastenhanced mrimaging of normal tissue and cancer, Radiology 237 (2005), 429-436.

[46] J.K. Litton and R.L. Theriault, Breast Cancer and pregnancy: Current concepts in diagnosis and treatment, Oncologist 15 (2010), 1238-1247.

[47] R. Vashi, R. Hooley, R. Butler, J. Geisel and L. Philpotts, Breast imaging of the pregnant and lactating patient: Imaging modalities and pregnancy-associated breast cancer, AJR AM J Roentgenol 200 (2013), 321-328.

[48] D. Taylor, J. Lazberger, A. Ives, E. Wylie and C. Saunders, Reducing delay in the diagnosis of pregnancy-associated breast cancer: How imaging can help us, $J$ Med Imaging Radiat Oncol 55 (2011), 33-42.

[49] E.J. Son, K.K. Oh and E.K. Kim, Pregnancy-Associated Breast Disease: Radiologic Features and Diagnostic Dilemmas, Yonsei Med J 47 (2006), 34-42.

[50] F. Rovera, F. Frattini, A. Coglitore, M. Marelli, S. Rausei, G. Dionigi, L. Boni and R. Dionigi, Breast Cancer in Pregnancy, Breast J 16 (Suppl.1) (2010), 22-25.

[51] A. Joshi and S. Maimoon, Limitations of fine needle aspiration cytology in subtyping breast malignancies - A report of three cases, J Cytol 24 (2007), 203-206.

[52] B. Karim, S. Ali, J. Landolfi, J. Mann, G. Liu, A. Christian, J. Dicello, D. Rosenthal and D. Huso, Cytomorphologic differentiation of benign and malignant mammary tumors in fine needle aspirate specimens from irradiated female SpragueDawley rats, Vet Clin Pathol 37 (2008), 229-236.

[53] A.M. Domanski, N. Monsef, H.A. Domanski, D. Grabau and M. Ferno, Comparison of the oestrogen and progesterone receptor status in primary breast carcinomas as evaluated by immunohistochemistry and immunocytochemistry: A consecutive series of 267 patients, Cytopathology 24 (2013), 21-25.

[54] G. Sizilio, C. Leite, A. Guerreiro and A.D. Neto, Fuzzy method for pre-diagnosis of breast cancer from the Fine Needle Aspirate analysis, Biomed Eng Online 11 (2012), 83. 
[55] R.M. Fearmonti, L.I. Batista, F. Meric-Bernstam, I. Bedrosian, H.M. Kuerer, K.K. Hunt, S.E. Singletary and G.V. Babiera, False negative rate of sentinel lymph node biopsy in multicentric and multifocal breast cancers may be higher in cases with large additive tumor burden, Breast J 15 (2009), 645-648.

[56] J. Jung, H. Park, J. Park and H. Kim, Accuracy of preoperative ultrasound and ultrasound-guided fine needle aspiration cytology for axillary staging in breast cancer, ANZ J Surg $\mathbf{8 0}$ (2010), 271-275.

[57] K. Turaga, A. Chau, J. Eatrides, J. Kiluk, N. Khakpour, C. Laronga and C. Leeb, Selective application of routine preoperative axillary ultrasonography reduces costs for invasive breast cancers, Oncologist 16 (2011), 942-948.

[58] S. Lale, K. Kure and D. Lingamfelter, Challenges to diagnose metaplastic carcinoma of the breast through cytologic methods: an eight-case series, Diagn Pathol 6 (2011), 7.

[59] J. Russo, G. Balogh and I.H. Russo, Breast cancer prevention, Climacteric 10 (Suppl.2) (2007), 47-53.

[60] C. Jeronimo, P. Monteiro, R. Henrique, M. Dinis-Ribeiro, I. Costa, V. Costa, L. Filipe, A. Carvalho, M. Hoque, I. Pais, C. Leal, M. Teixeira and D. Sidransky, Quantitative hypermethylation of a small panel of genes augments the diagnostic accuracy in fine-needle aspirate washings of breast lesions, Breast Cancer Res Treat 109 (2008), 27-34.

[61] M.L. Talman, B.B. Rasmussen, J. Andersen and I.J. Christensen, Estrogen Receptor analyses in the Danish Breast Cancer Cooperative Group. History, methods, prognosis and clinical implications, Acta Oncol 47 (2008), 789-794.

[62] D.M. Harvell, J. Kim, J. O'Brien, A.C. Tan, V.F. Borges, P. Schedin, B.M. Jacobsen and K.B. Horwitz, Genomic signatures of pregnancy-associated breast cancer epithelia and stroma and their regulation by estrogens and progesterone, Horm Canc 4 (2013), 140-153.

[63] M.E. Hammond, D.F. Hayes, M. Dowsett, D.C. Allred, K.L. Hagerty, S. Badve, P.L. Fitzgibbons, G. Francis, N.S. Gold- stein, M. Hayes, D.G. Hicks, S. Lester, R. Love, P.B. Mangu, L. McShane, K. Miller, C.K. Osborne, S. Paik, J. Perlmutter, A. Rhodes, H. Sasano, J.N. Schwartz, F.C. Sweep, S. Taube, E.E. Torlakovic, P. Valenstein, G. Viale, D. Visscher, T. Wheeler, R.B. Williams, J.L. Wittliff and A.C. Wolff, American Society of Clinical Oncology/College of American Pathologists guideline recommendations for immunohistochemical testing of estrogen and progesterone receptors in breast cancer, Arch Pathol Lab Med 134 (2010), 907-922.

[64] A. Singh, J.S. Nigam, V. Misra and P.A. Singh, Diagnosis of a nonpalpable intraductal papilloma without radiological abnormality by nipple discharge smear examination: A case report, Breast Cancer: Basic and Clinical Research 8 (2014), 69-72.

[65] W.Y. Lee, Cytology of abnormal nipple discharge: A cytohistological correlation, Cytopathology 14 (2003), 19-26.

[66] J.M. Faupel-Badger, K.F. Arcaro, J.J. Balkam, H.A. Eliassen, F. Hassiotou, C.B. Lebrilla, K.B. Michels, J.R. Palmer, P. Schedin, A.M. Stuebe, C.J. Watson and M.E. Sherman, Postpartum remodeling, lactation, and breast cancer risk: Summary of a national cancer institute-sponsored workshop, $J$ Natl Cancer Inst 105 (2013), 166-174.

[67] P. Mills, A. Sever, J. Weeks, D. Fish, S. Jones and P. Jones, Axillary ultrasound assessment in primary breast cancer: An audit of 653 cases, Breast J 16 (2010), 460-463.

[68] B.J. Van Wely, J.H.W. De Wilt, P.J.C. Schout, B. Kooistra, C.A.P. Wauters, D. Venderinck and L.J.A. Strobbe, Ultrasound-guided fine-needle aspiration of suspicious nodes in breast cancer patients; selecting patients with extensive nodal involvement, Breast Cancer Res Treat 140 (2013), 113118.

[69] A. Keleher, R. Wendt, E. Delpassand, A.M. Stachowiak and H.M. Kuerer, The safety of lymphatic mapping in pregnant breast cancer patients using tc-99m sulfur colloid, Breast $J \mathbf{1 0}$ (2004), 492-495. 